\title{
Novel mass hierarchy and discrete excitation spectra from quantum-fluctuating D-branes
}

\author{
G. K. Leontaris \\ Theoretical Physics Division, Ioannina University, GR-45110 Ioannina, Greece \\ N. E. Mavromatos \\ CERN, Theory Division, 1211 Geneva 23, Switzerland \\ and Theoretical Physics Group, Department of Physics, King's College London, Strand, London WC2R 2LS, United Kingdom
}

(Received 14 November 2000; published 11 June 2001)

\begin{abstract}
We elaborate further on a recently proposed scenario for generating a mass hierarchy through quantum fluctuations of a single D3-brane, which represents our world embedded in a bulk five-dimensional space-time. In this scenario, the quantum fluctuations of the D3-brane world in the bulk direction, quantified to leading order via a "recoil", world-sheet logarithmic conformal field theory approach, result in the dynamical appearance of a supersymmetry breaking (obstruction) scale $\alpha$. This may be naturally taken to be at the TeV range, in order to provide a solution to the conventional gauge-hierarchy problem. The bulk spatial direction is characterized by the dynamical appearance of a horizon located at $\pm 1 / \alpha$, inside which the positive energy conditions for the existence of stable matter are satisfied. To ensure the correct value of the four-dimensional Planck mass, the bulk string scale $M_{s}$ is naturally found to lie at an intermediate energy scale of $10^{14} \mathrm{GeV}$. As an exclusive feature of the D3-brane quantum fluctuations ("recoil") we find that, for any given $M_{5}$, there is a discrete mass spectrum for four-dimensional Kaluza-Klein (KK) modes of bulk graviton and/or scalar fields. KK modes with masses $0 \leqslant m<\sqrt{2} \alpha \ll M_{s}$ are found to have wave functions peaked, and hence localized, on the D3-brane at $z=0$.
\end{abstract}

DOI: 10.1103/PhysRevD.64.024008

\section{INTRODUCTION}

In recent years considerable interest has been concentrated on exploring the idea that space-time is actually (4 $+n$ ) dimensional, with the four-dimensional observable world being a membrane (Dirichlet brane [1]) of some string theory living in the $(4+n)$-dimensional bulk space-time [2]. The literature is growing rapidly, and many interesting models have been considered, some of which appear to have falsifiable predictions in the next-generation accelerators, such as the Fermilab Tevatron and CERN Large Hadron Collider (LHC). In some of these models, the extra (bulk) dimensions are taken to be relatively large, compared to the traditional Planck scale, implying, for instance, a bulk gravitational scale at the range of a few $\mathrm{TeV}$. In the case of compact extra dimensions there are induced modifications of the four-dimensional Newton law, which may become phenomenologically important for $\mathrm{TeV}$ scale gravity [3]. In this scenario, the experimental success of the inverse-square law of Newton seemed to imply precisely four non-compact dimensions only.

However, the work of Ref. [4] has demonstrated that the situation is completely different in cases where the higherdimensional metric was not factorizable, namely the case where there is a warp factor in front of the four-dimensional metric which depends on the coordinates of the bulk extra dimensions. According to this approach, our universe is a static flat domain-wall which, in the simplest case of five dimensions, separates two regions of five-dimensional anti-de Sitter (AdS) space-time. In its simplest version [4], the scenario is realized by introducing a positive energy brane at the origin and a negative energy brane at distance $z$ where our world is located and where the graviton amplitude is exponentially suppressed. Modifications to the above pic-
PACS number(s): 04.50.+h, 11.10.Kk, 98.80.Cq

ture with positive energy branes allowing also the possibility of infinite extra dimensions, multi-brane solutions, and supergravity embedded versions were considered in the literature [5-7]. Thus, it is worth noticing that the bulk dimensions are not necessarily compact. The rather important point of [4], however, was the demonstration of the localization of the bulk gravitational fluctuations on the three-dimensional brane, which plays the role of our world. This localization property was demonstrated by mapping the problem of the dynamics of these fluctuations into a one-dimensional Schrödinger eigenvalue problem.

A characteristic feature of such models was the presence of a massless mode for the graviton (in agreement with Lorentz covariance on the brane) together with a continuum of massive Kaluza-Klein (KK) states on the four-dimensional world. These KK modes have different properties as compared with the factorizable case. The presence of such KK states leads to corrections of the four-dimensional Newton's law; such corrections, however, are suppressed by quadratic powers of the inverse Planck mass scale, and hence are unobservable for all practical purposes. As a result of the above localization, a solution to the mass hierarchy emerges in the sense that the weak scale is generated from a large scale of the order of Planck mass through an exponential hierarchy, induced by the presence of the warp factor in the metric of the four-dimensional world.

In the original scenario of [4] only fields in the gravitational multiplet have been allowed to propagate in the bulk direction. The standard model fields were assumed to live exclusively on the brane worlds. In the approach of [6], however, standard model matter fields of mass close to the string scale $M_{s}$, which is a natural mass scale in the bulk, were assumed to exist also in the bulk, where they can propagate normally. Within the context of the Randall-Sundrum space 
time [4], then, it was shown that the KK modes of such bulk fields are characterized by four-dimensional masses which are also exponentially suppressed, thereby implying a novel mass hierarchy.

In both models $[4,6]$ one requires the existence of a second brane, and the distance between them is supposed to be determined dynamically by the minimization of a particular potential for the so-called modulus (radion) field. This minimization is argued to provide a stabilization mechanism for the radion [8].

\section{QUANTUM FLUCTUATING (SINGLE) BRANES AND NON-FACTORIZABLE BULK METRICS}

However, in all the above approaches quantum fluctuations of the branes have been ignored, given that the latter have been assumed rigid. For consistency with general relativity, such an assumption must be relaxed, if one is dealing with finite-mass solitonic stringy structures. In a previous paper [9], we have made an attempt to discuss the incorporation of such effects in a single D3-brane, embedded in a five-dimensional geometry. A single brane situation may be thought of as the limiting case where any other brane has run away to infinity (i.e. to a bulk distance much larger than any other distance scale in the problem).

We have found that the quantum fluctuations of the D3brane, which can be described by the excitation of (open and closed) strings [10-12], formulated in terms of logarithmic conformal field theory on the world-sheet [13], results in a distortion of the surrounding space time [11,9], in such a way that the five-dimensional invariant line element corresponding to the induced bulk metric is of non-factorizable form between the bulk $z$ and D3-brane coordinates $X^{I}$.

$$
\begin{aligned}
d s_{f}^{2} & =e^{-2 \sigma(z)} g_{I J}\left(X^{K}\right) d X^{I} d X^{J}-d z^{2}, \\
\sigma(z) & =-\frac{1}{2} \ln \left(\left|1-\alpha^{2} z^{2}\right|\right) .
\end{aligned}
$$

In the above formula $\alpha$ denotes the width of the momentum fluctuations of the D3-brane in the bulk direction. ${ }^{1}$

It is important to notice that, as a result of the requirement of convergence of the world-sheet path integrals, the fourdimensional (brane) space time, with metric $g_{I J}$ is assumed to be Euclidean. Passage to a Minkowskian space-time takes place only at the very end of the computations, by means of analytic continuation in the target time. In the leading-order approach of Ref. [9] to the D3-brane recoil, the fourdimensional metric $g_{I J}$ is found to be flat.

In the approach of [12], the width $\alpha$ can be computed to leading order in a weak string coupling by an appropriate resummation procedure over pinched annuli on the worldsheet, with the result

\footnotetext{
${ }^{1}$ However, as discussed in [9], an alternative scenario exists in which $\alpha$ is related to the momentum transfer during the collision of a macroscopic D1-brane (string), propagating along the bulk direction, with the D3-brane world at a certain time $t_{0}$ in the remote past.
}

$$
\alpha \sim g_{s} \Delta P
$$

where $g_{s} \ll 1$ is the string coupling, which is assumed weak enough so that the world-sheet perturbation theory is valid, and $\Delta P$ is the momentum uncertainty (along the bulk direction) of the D3-brane. The latter quantity is bounded from below as a result of the stringy uncertainty principle, which, to a leading order approximation for weakly coupled branes, has been derived in [12] using logarithmic world-sheet conformal field theory methods [13].

At present the precise value of $\alpha$ is unknown, given that this would require knowledge of the wave function of the five-dimensional brane model, which is unknown. However, following analogous situations in quantum mechanics, one may assume a saturation of the uncertainty principle, which allows $\Delta P$ to be expressed in terms of the minimum length $\Delta z$. In the case at hand, within the world-sheet weakly coupled string model, the latter is found to be [12]

$$
\Delta z \sim \frac{g_{s}^{\eta / 2}}{M_{s}}
$$

where $M_{s}$ is the bulk string scale. The quantity $\eta$ is associated with modular infinities in the $\sigma$-model genus expansion [12], and currently cannot be computed within the $\sigma$-model perturbation theory. Its value may be fixed by the requirement that the minimum length (3) coincides with the one obtained from generic kinematical arguments in brane theory [14], which implies $\eta=2 / 3$. Standard string theory would require $\eta=0$. In general, for our purposes in this paper, one may leave at this stage $\eta$ as an unknown parameter to be bounded by the current phenomenology.

From Eqs. (3) and (2), then, one obtains

$$
\alpha \sim g_{s}^{1-\eta / 2} M_{s} .
$$

The non-factorizable metric (1) results in the following modified Einstein term in the action [4]:

$$
S=\int d z e^{-2 \sigma(z)} \int d^{4} X R^{(4)}[g]+\cdots
$$

where the superfix (4) denotes four-dimensional quantities, depending only on the $X^{I}, I=1, \ldots 4$ coordinates. Equation (5) implies that the effective four-dimensional Planck's constant, for an observer living on the D3-brane, is

$$
M_{P}^{2}=M_{s}^{3} \int d z e^{-2 \sigma(z)} .
$$

In Ref. [9] we have demonstrated the dynamical formation of horizons, located at $z= \pm 1 / \alpha$, which effectively restrict the bulk space time inside $-1 / \alpha<z<1 / \alpha$. In the context of the models of Ref. [6] in which matter fields are allowed to propagate in the bulk, the role of horizons can be understood simply by considering the positive energy conditions $[15,16]$ for the (Minkowskian version of the) space time (1). It suffices to examine the weakest of these conditions, which can be formulated in terms of the stress-energy tensor $T_{\mu \nu}$ as follows: 


$$
T_{\mu \nu} \zeta^{\mu} \zeta^{\nu} \geqslant 0, \quad \mu, \nu=1, \ldots 5
$$

where $\zeta^{\mu}$ are arbitrary null vectors.

Using Einstein's equations, one may replace the stressenergy tensor by the corresponding Einstein tensor in the geometry (1). It is easy to see, then, that the weakest energy condition amounts simply to the requirement

$$
\sigma(z)^{\prime \prime} \geqslant 0 .
$$

If the weakest energy condition is violated, all the others are violated too. From Eq. (1) we observe [9] that the weakest energy condition (8) is satisfied only inside the horizon $-1 / \alpha<z<1 / \alpha$. This implies that stable matter can only exist inside this region of the bulk space time $[15,16]$.

In view of this result we then define the effective fourdimensional Planck (6) mass in our model of a single fluctuating D-brane world as

$$
\begin{aligned}
M_{P} & =M_{s}^{3 / 2}\left(\int_{-1 / \alpha}^{1 / \alpha} d z e^{-2 \sigma(z)}\right)^{1 / 2} \sim \frac{2}{\sqrt{3}} M_{s}\left(\frac{M_{s}}{\alpha}\right)^{1 / 2} \\
& =\frac{2}{\sqrt{3}} \frac{M_{s}}{g_{s}^{(2-\eta) / 4}}
\end{aligned}
$$

where we used Eq. (4).

The analysis of [9] has demonstrated that the space-time (1) satisfies Einstein's equations, provided one includes an excitation energy term of a "stack" of parallel D3-branes inside the horizon region, described by a four-dimensional term

$$
\int d z \int d^{4} x \sqrt{g} V(z)
$$

where the flat-space integral denotes the continuum limit of the sum representing the (infinite) stack of parallel D3-branes in the region $|z|<1 / \alpha$.

As discussed in [9] in some detail, any D3-brane in the "stack," say at a position $z_{i}$ along the bulk $z$ direction, is characterized by a finite tension, call it $T\left(z_{i}\right)$. By assuming a density of D3-branes $\rho(z)$ in the continuous description of the stack, which is a satisfactory approximation for our purposes here, one obtains that the sum of the D3-brane tensions $T\left(z_{i}\right)$ in the stack, which would describe their contribution to the excitation energy, may be replaced by an appropriate integral:

$$
\sum_{i} \int d^{4} x \sqrt{g} T\left(z_{i}\right) \rightarrow \int d z \int d^{4} x \sqrt{g} \rho(z) T(z)
$$

where the index $i$ runs over D3-branes in the stack. The product $\rho(z) T(z) \equiv V(z)$ is what contributes to the vacuum energy (10), in a Lorentz-noninvariant way in the bulk, which is a natural consequence of the presence of the "stack" of the parallel D3-branes. The precise form of the quantity $V(z)$ is determined by consistency with Einstein's equations of motion, or, equivalently, conformal invariance conditions of the underlying $\sigma$-model, as discussed in great detail in [9].

This configuration describes formally the quantum fluctuations of a single D3 brane inside the horizon [9]. It is important to notice that the term (10) does not vary with respect to the fifth component of the metric, unlike the corresponding bulk cosmological "constant" $\Lambda$ term. As already mentioned, the explicit dependence of $V(z)$ on the $z$ coordinate in general breaks Lorentz invariance in the bulk, but this is not unexpected as a result of the presence of the fluctuating D-brane. On the other hand, Lorentz invariance remains a good symmetry on the D3-brane. ${ }^{2}$

The result for $V(z)$, that solves Einstein's equations in the five-dimensional geometry, is

$$
\begin{aligned}
\frac{V(z)}{12 M_{s}^{3}}= & \frac{1}{2} \frac{\alpha^{2}}{(1+\alpha z)^{2}}+\frac{1}{2} \frac{\alpha^{2}}{(1-\alpha z)^{2}} \\
& -\frac{\alpha^{2}}{|1+\alpha z|} \delta(1+\alpha z)-\frac{\alpha^{2}}{|1-\alpha z|} \delta(1-\alpha z) .
\end{aligned}
$$

At $z=0$, which was the equilibrium location of the D3-brane world, the excitation energy is positive $V(z)=12 \alpha^{2}$. On the other hand, the space-time (1) is characterized by a "vacuum energy" $\Lambda(z)$

$$
\frac{\Lambda}{24 M_{s}^{3}}=-\left[\sigma^{\prime}(z)\right]^{2}
$$

which vanishes at $z=0$, and is negative for $z \neq 0$ (anti-de Sitter type bulk).

As discussed in [17], the result (12) and (13) would signal supersymmetry obstruction [18] on the original D3-brane as a result of the quantum recoil fluctuations: the excited (fluctuating) state of the brane is not supersymmetric, due to a non-vanishing excitation energy $V(0) \sim 12 \alpha^{2}$, while the ground state (not recoiling branes) could be, as a result of the anti-de Sitter type geometry (13). The scale of this supersymmetry obstruction would be set by $\alpha$ then, which in view of Eqs. (4), (9), would define a new hierarchy for weakly coupled strings $g_{s} \ll 1$.

From Eq. (4), one finds the following hierarchy of scales:

$$
M_{s} \sim\left(\frac{3}{4} \alpha M_{P}^{2}\right)^{1 / 3} .
$$

Indeed, for $M_{P} \sim 10^{19} \mathrm{GeV}$ and $\mathcal{O}(1) \mathrm{TeV}<\alpha$ $\leqslant \mathcal{O}(100) \mathrm{TeV}$, as appears to be necessary for a solution to the gauge hierarchy problem on the D3 world, one would obtain, from Eq. (14),

\footnotetext{
${ }^{2}$ More general models of foam, characterized by intersecting branes, or D3-branes "punctured"' by D-particle defects also exist [16], but we shall not deal with such models here.
} 


$$
M_{s} \sim 10^{14} \mathrm{GeV}
$$

implying an intermediate string scale. In this case, the hierarchy (14) should be compared with the one encountered in a class of conventional-field-theory models involving intermediate-scale $M_{I}$ unification, $M_{I} \sim \sqrt{M_{P} m_{W}}$, where $m_{W}$ is the electroweak scale.

Notice that, in contrast to the model of [4] involving two parallel branes separated by a distance $r_{c}$, which sets the hierarchy in that case, our model has a single brane, whose quantum (momentum) fluctuations in the extra dimension are responsible for generating a hierarchy (14) between the supersymmetry-breaking-scale and the string (or Planck) mass scale (9).

Notice also, that in case one assumes saturation of the uncertainty principle, determining $\alpha$, then from Eqs. (4) and (9) we would obtain

$$
g_{s}^{1-\eta / 2} \leqslant 10^{-9},
$$

implying fairly weak string couplings $\left[g_{s} \sim 10^{-9}\right.$ for $\eta=0$ (ordinary strings), or $g_{s} \sim 10^{-13}$ for $\eta=2 / 3$ (branes)]. Such weak couplings are not unusual in string theories dual to ordinary string theories [19]. However, as we have explained above, for our purposes here we prefer to treat $\alpha$ as a phenomenological (but, in principle, theoretically calculable) parameter, to be constrained by the current data.

Some clarifying comments are in order at this point. Although the string coupling (16) that yields the correct hierarchy is small, and one may think of it as a finely tuned parameter, however as we have explained above, the interesting issue is whether such weak couplings can arise in a dynamical way from the underlying string theory itself. For instance, in our approach, the scale $\alpha$ expresses the uncertainty in the momentum fluctuations (along the bulk $z$ direction) of the D-brane, and as such it is a dynamical quantity that may be determined in principle, if sufficient knowledge on the pertinent wave function were available. Unfortunately, within the present technology, this is not feasible. However, as we have already remarked, such weak couplings do appear in certain dual theories of strings [19], and in our approach such couplings are not necessarily associated directly with phenomenological gauge couplings on the D3brane world. Notably, a string coupling (16) $g_{s} \sim e^{-2\langle\Phi\rangle}$ $\sim 10^{-13}$, may be related to a dilaton $\operatorname{VEV}\langle\Phi\rangle \sim \mathcal{O}(10)$, which may not be unnatural in our recoiling D-brane context. At any rate, much more research is necessary before firm conclusions are reached on this important issue, but we believe that the above approach is novel enough to justify further studies along such directions.

\section{LOCALIZATION OF FIELDS ON THE FLUCTUATING D3-BRANE WORLD AND A NOVEL MASS HIERARCHY}

\section{A. Graviton modes}

An interesting result of the analysis in [9] was the demonstration of the localization of graviton four-dimensional KK modes with masses $m<\sqrt{2} \alpha$ on the D3-brane, with the simultaneous expulsion of modes with masses higher than $\sqrt{2} \alpha$ on the horizons. To show this, we followed [4] and used the following ansatz for separating variables $X^{I}$ and $z$, as far as (small) quantum fluctuations of the bulk graviton state $\hat{h}\left(X^{I}, z\right)$ about the background (1) are concerned:

$$
\hat{h}\left(X^{I}, z\right)=\lambda(z) e^{i p_{I}^{E} X^{I}}
$$

where the notation $p_{I}^{E}$ in the momenta on the brane has been explicitly stated to remind the reader that we are working on a Euclidean setup for $\left\{X^{I}\right\}$, and hence for massive KK excitations, of mass squared $m^{2}>0$. The on-shell condition for these modes should then read

$$
\left(p_{I}^{E}\right)^{2}=-m^{2}<0
$$

The equation for such small fluctuations, can be obtained by linearizing Einstein's equations around the AdS background (1) and choosing an appropriate gauge for the fluctuations of the metric. Upon introducing the ansatz (17), and using Eq. (18), the equation becomes a one-dimensional Schrödingertype eigenvalue equation for the bulk modes $\lambda(z)[4,9]$

$$
-\lambda^{\prime \prime}(z)+\left[4\left(\sigma^{\prime}\right)^{2}-2 \sigma^{\prime \prime}\right] \lambda(z)=-m^{2} e^{2 \sigma} \lambda(z) .
$$

It is important to note the minus sign in front of the mass term on the right-hand side of Eq. (19), which is due to the Euclidean nature of $X^{I}$ hyperplane (18). As we have explained in [9], this plays the important physical role of inducing localization of light KK modes with masses below a critical mass $m_{c r}=\sqrt{2} \alpha$.

To see this, we recall that Eq. (19) has the form of a one-dimensional Scrhödinger's equation, with potential

$$
\begin{aligned}
\mathcal{V}(z)= & -\left(\frac{2 \alpha^{2}-m^{2}}{\left|1-\alpha^{2} z^{2}\right|}-\frac{\alpha^{2}}{|1+\alpha z|} \delta(1+\alpha z)\right. \\
& \left.-\frac{\alpha^{2}}{|1-\alpha z|} \delta(1-\alpha z)\right)
\end{aligned}
$$

which is depicted in Fig. 1. It is important to notice that this potential has non-trivial $\delta$-function (repulsive) boundaries, which imply a dynamical restriction of the bulk space time inside the region $\alpha^{2} z^{2}<1$.

For the region close to the brane $z=0$, we may ignore the effect from the boundaries. Notice that near $z=0$ the potential is attractive for

$$
m^{2}<2 \alpha^{2}
$$

which is the origin of the critical mass for KK excitations of massless bulk fields (including gravitons) [9]. The potential (cf. Fig. 1) starts as a inverted harmonic oscillator potential [20] around $z=0$, but then deviates significantly from it (by anharmonic terms), to end up in $\delta$-function opaque (repulsive) walls at $z= \pm 1 / \alpha, \mathcal{V}( \pm 1 / \alpha) \sim \Omega \delta(0)$ with infinite opacity $\Omega=\operatorname{Lim}_{z \rightarrow \mp 1 / \alpha}(1 /|1 \pm \alpha z|)$. Therefore the wave function should vanish at the boundaries, thus effectively implying a dynamical restriction of the bulk direction inside 


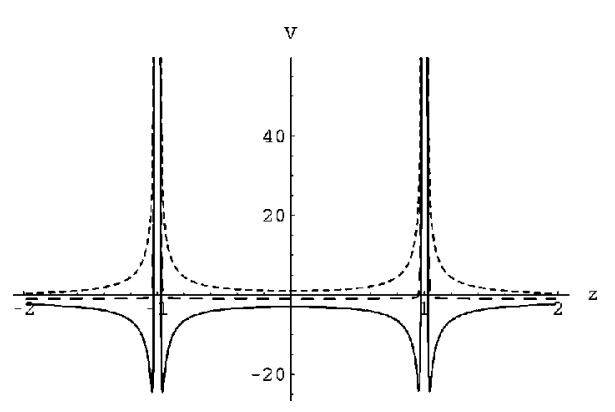

FIG. 1. Schematic representation of the "suspension bridge", potential of the equivalent Schrödinger equation corresponding to the dynamics of a quantum-fluctuating D3-brane in the bulk direction of a five-dimensional space time. The solid curve corresponds to the massless Kaluza-Klein (KK) four-dimensional mode, while the dashed curves represent the potential for the KK modes with masses $m^{2} \geqslant m_{c r}=2 \alpha^{2}$. The wave functions of these modes are no longer localized on the D3-brane at $z=0$. The reader is invited to notice the completely impenetrable potential walls (denoted in the figure by disjoint lines), which is the result of the D3-brane bulk quantum fluctuations ("recoil").

the region $z^{2} \alpha^{2}<1$. This may be seen as another alternative to compactification [9], physically different from the one proposed in [4].

We now note that away from the boundaries the Schrödinger equation can be solved analytically. Requiring $\left.(d \lambda / d z)\right|_{(z=0)}=0$, the graviton states wave function is given by

$$
\begin{aligned}
\lambda(z) \sim & { }_{2} F_{1}\left(-\frac{1}{4}-\frac{3}{4}\left[1-\left(\frac{2 m}{3 \alpha}\right)^{2}\right]^{1 / 2},\right. \\
& \left.-\frac{1}{4}+\frac{3}{4}\left[1-\left(\frac{2 m}{3 \alpha}\right)^{2}\right]^{1 / 2}, \frac{1}{2},(\alpha z)^{2}\right) .
\end{aligned}
$$

From the behavior of the hypergeometric function, we find that states with mass less than the critical value $m$ $<\sqrt{2} \alpha$ have wave functions which are peaked on the D3brane world at $z=0$. If one, now, includes the $\delta$-function boundaries, then the resulting wave function is localized inside the region $\alpha^{2} z^{2}<1$ [9]. The massless KK graviton mode $(m=0)$ is depicted in Fig. 2, where the effect of the boundaries at $z= \pm 1 / \alpha$ in Eq. (20) is included. For the critical value $m=m_{c r}$ we have $\lambda_{c r}(z)={ }_{2} F_{1}\left[-\frac{1}{2}, 0, \frac{1}{2},(\alpha z)^{2}\right]=1$, corresponding to a constant mode (with amplitude normalized to one for convenience), which is thus not compatible with the vanishing boundary conditions at $z= \pm 1 / \alpha$, imposed by the opaque walls. We remind the reader that proper inclusion of the boundary effects in the Schrödinger equation (19) requires numerical treatment. From such an analysis, we deduce [9] that, as a result of the $\delta$-functions in the corresponding potential, states with $\mathrm{KK}$ masses slightly below the critical value, $m \sim \sqrt{2} \alpha \pm \epsilon, \epsilon \rightarrow 0^{+}$, are distorted due to the boundary effects at $z= \pm 1 / \alpha$, and hence begin to delocalize.

On the other hand, the wave functions of modes with masses $m>m_{c r}$ are infinitely peaked at the boundaries $z=$ $\pm 1 / \alpha[9]$, and hence they are unbounded, and could be dis- carded on physical grounds. ${ }^{3}$ The above features are easily deduced by the analogy of the corresponding Schrödinger potential for the case $m>m_{c r}$, near $z=0$, with that of an ordinary harmonic oscillator. As can be readily seen, such a supercritical case would correspond to a negative-energy eigenvalue state, which does not exist for an oscillator potential with a positive minimum.

The presence of massive $\mathrm{KK}$ modes for the graviton on the D3-brane world leads [9] to modifications of Newton's law on the brane hypersurface $\left\{X^{I}\right\}, I=0, \ldots 3$ at $z=0$, due to the exchange of massive modes with masses up to $\sqrt{2} \alpha$. As discussed in [9], in the case of free boundary conditions on the horizons $|z|=1 / \alpha$, there are (attractive) corrections to Newton's law of $r^{-2}$ scaling, which are suppressed by a power of $\alpha / M_{s}$. Notice that on account of the hierarchy (14), for supersymmetry-breaking scales $\alpha \leqslant 100 \mathrm{TeV}$, as required for a solution to the gauge hierarchy problem, and a string scale $M_{s} \sim 10^{14} \mathrm{GeV}$, the corrections to Newton's law are negligible, consistent with the current phenomenology.

On the other hand, if one imposes periodic boundary conditions for the various mode wave functions on the horizons, which corresponds to compactification, then the KK spectrum is discrete with masses $m=n \alpha / 2, n=0,1,2, \ldots$. In this case one essentially identifies the two horizons, and thus one has effectively a two brane scenario. In view of Eq. (21), only the first few $(n=0,1,2)$ of these KK modes are localized on the D3 brane. This implies that the corresponding corrections to Newton's law involve a sum over these few modes:

$$
V_{G}=-\frac{G_{N} m_{1} m_{2}}{r}\left(1+\sum_{n=1}^{2}\left(\frac{M_{P}^{2}}{c_{n}^{2}}\right) e^{-(n / 2) \alpha r}\right)
$$

where $c_{n}$ are the corresponding couplings, $G_{N}=1 / M_{P}^{2}$ is the four-dimensional Newton's constant, and $M_{P}$ is the fourdimensional Planck mass. For our case of $\alpha \leqslant 100 \mathrm{TeV}$, again such corrections are negligible for distances down to submillimeter scales, which is the current experimental accuracy for such tests.

\section{B. Massive scalar fields}

Next we examine the scenario of Ref. [6] for matter bulk fields within the context of the present model (1). To this end we consider an expansion of a bulk scalar field in terms of "'modified plane waves,' to take proper account of the induced curved metric (1):

$$
\Phi\left(X^{I}, z\right)=\frac{1}{g^{1 / 4}} e^{i P_{I} X^{I}} \varphi(z)=e^{i P_{I} X^{I}} e^{2 \sigma(z)} \varphi(z) .
$$

Following the approach of [6], we consider the action of a bulk matter scalar field, of five-dimensional mass $M_{5}$, propa-

\footnotetext{
${ }^{3}$ Even if one accepts unbounded solutions to the original $\mathrm{KK}$ mode problem, such modes are definitely not localized on our brane world at $z=0$, and from this point of view are of no interest to us here.
} 

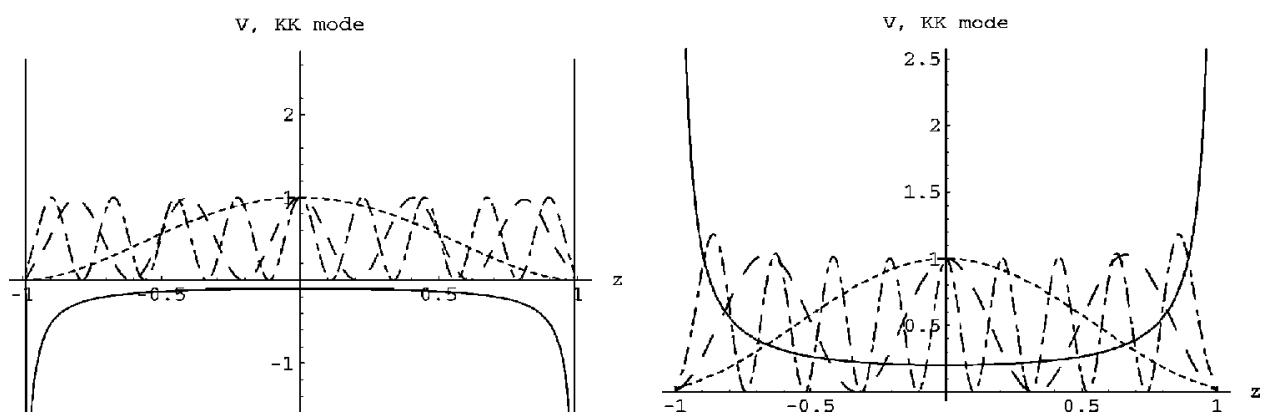

FIG. 2. Field localization in Euclidean space times. The figure on the left shows the modulus squared of the scalar-field wave functions (arbitrarily normalized) for three light Kaluza-Klein (KK) modes, with masses below the critical mass $0 \leqslant m<m_{c r}=\sqrt{2} \alpha$. The continuous line corresponds to the potential of the associated Schrödinger equation. The dotted curve denotes the massless $M_{5}=0$ case (which is formally the same as the graviton case). The dashed and dashed-dotted lines, on the other hand, correspond to $M_{5} \neq 0$ cases. The higher the value of $M_{5}$, the larger the number of nodes of the wave function. The wave functions corresponding to these modes are peaked on the $z$ $=0$ brane world and vanish at the boundaries $z= \pm 1 / \alpha$. The figure on the right shows the modulus squared of the wave functions of three heavy KK modes with masses $m>m_{c r}=\sqrt{2} \alpha$. The continuous line denotes again the corresponding Schrödinger potential. The dotted line corresponds to the case $M_{5}=m$, and exhibits localization on the D3 brane at $z=0$. The dashed curve corresponds to the case $M_{5}<m$, and has a local peak at $z=0$, while vanishing at the boundaries. Finally, the dashed-dotted curve corresponds to the case $M_{5}>m$. The wave functions of these latter modes peak near the boundaries, but vanish exactly at the boundaries due to the infinite opacity.

gating inside the horizon region of the (Euclidean) space time (1), where such matter can live in a stable form due to the energy conditions (8):

$$
\begin{aligned}
S= & \frac{1}{2} \int d^{4} x \int_{-1 / \alpha}^{1 / \alpha}\left[-e^{-2 \sigma(z)} \delta^{I J} \partial_{I} \Phi \partial_{J} \Phi\right. \\
& \left.+\Phi \partial_{z}\left(e^{-4 \sigma(z)} \partial_{z} \Phi\right)+M_{5}^{2} e^{-4 \sigma(z)} \Phi^{2}\right] .
\end{aligned}
$$

Upon inserting the decomposition (24), and using the fourdimensional on-mass-shell (Euclidean) condition $\left(P_{I}^{E}\right)^{2}=$ $-m_{\mathrm{sc}}^{2}<0$, we arrive at a one-dimensional Schrödinger-like equation for the KK mode wave functions $\varphi(z)$ :

$$
-\varphi^{\prime \prime}(z)+\left[4\left(\sigma^{\prime}\right)^{2}-2 \sigma^{\prime \prime}\right] \varphi(z)=-\left(m_{\mathrm{sc}}^{2} e^{2 \sigma}-M_{5}^{2}\right) \varphi(z)
$$

which for massless bulk scalar modes $M_{5}$ is identical to that for the graviton KK modes (19), leading to localization on the D3-brane of modes lighter than $\sqrt{2} \alpha$.

For massive scalar bulk fields, ${ }^{4}$ with $M_{5} \neq 0$, the Schrödinger equation (26) becomes (inside the horizon)

$$
\begin{gathered}
-\varphi^{\prime \prime}(z)-\left(\frac{2 \alpha^{2}-m_{\mathrm{sc}}^{2}}{\left|1-\alpha^{2} z^{2}\right|}-\frac{\alpha^{2}}{|1+\alpha z|} \delta(1+\alpha z)\right. \\
\left.-\frac{\alpha^{2}}{|1-\alpha z|} \delta(1-\alpha z)+M_{5}^{2}\right) \varphi(z)=0 .
\end{gathered}
$$

There are rigorous theorems on the existence of solutions of this equation [22]. Inside the region of interest, $\alpha^{2} z^{2}<1$, away from the boundaries, where the effects of the $\delta$-functions can be ignored, the latter can be expressed as appropriate sums of Bessel and/or hypergeometric functions.

\footnotetext{
${ }^{4} \mathrm{~A}$ similar analysis is valid for massive vector bulk fields.
}

For generic values of the parameters the solutions are not known in a closed form, but only recursion relations among the summation coefficients have been provided [22]. For the purposes of our work here, we therefore restrict ourselves to a numerical analysis of the solutions, including the boundary terms.

Note that in the massless limit $M_{5}=0$ the equation becomes similar to Eq. (19). The potential corresponding to Eq. (27) has analogous form to that in Fig. 1, the only difference being the positive shift by the constant mass term $M_{5}^{2} \neq 0$. Such terms may be viewed as providing energy-eigenvalue terms in the corresponding Schrödinger equation.

The potential is attractive, in the interior $\alpha^{2} z^{2}<1$, only for four-dimensional KK modes of the bulk massive (scalar) fields for which Eq. (21) is satisfied. Such KK modes have wave functions which peak at $z=0$. The above conclusion is supported by a numerical analysis analogous to the one in the graviton case (see Fig. 2, which depicts the relevant probability densities for various cases). The modes that violate Eq. (21), on the other hand, correspond to a Schrödinger potential which is similar to that of an ordinary harmonic oscillator near $z=0$ (cf. Fig. 1), but deviates significantly from it by anharmonic terms as one goes to higher values of $z$ to end up in two infinitely opaque walls at $z= \pm 1 / \alpha$.

The energy spectrum is discrete, given that the situation is equivalent to that of a particle bound inside a potential well with infinitely opaque walls. ${ }^{5}$ We note that the discreteness of the spectrum is a generic feature of the recoil-induced

\footnotetext{
${ }^{5} \mathrm{We}$ stress that this is an exclusive feature of the completely impenetrable potential-wall terms $[1 /(1 \pm \alpha z)] \delta(1 \pm \alpha z)$, induced by the D3-brane recoil. If only $\delta$-function terms were present, as in the conventional D-brane-inspired models of [4], where recoil is ignored, then there would be a finite possibility of wall penetration, in which case one would also have continuous energy spectra, depending on the value of the energy.
} 
space-times with anti-de Sitter like bulk [23], such as Eq. (1). One therefore may expect nodes in the wave function, whose number depends on the value of the energy $M_{5}^{2}(\mathrm{Fu}-$ bini's theorem [24]). This is confirmed by a numerical analysis of Eq. (27). The discreteness of the energy spectrum is interesting in that it implies discrete allowed values for the bulk mass $M_{5}^{2}$ in terms of the quantity $\left|m^{2}-2 \alpha^{2}\right|$, and hence quantization of $m$ for given $M_{5}, \alpha$.

Unfortunately, for generic values of the parameters the corresponding spectrum cannot be obtained analytically. However, one may obtain approximate solutions in certain cases, which capture the qualitative features. For instance, in the massless case $M_{5}=0$, discussed in the previous subsection, away from the boundaries, the Schrödinger equation becomes hypergeometric (22). In the massive case, $M_{5} \neq 0$, and for modes $m^{2}<2 \alpha^{2}$ one may consider the Schrödinger equation (27) for $\alpha^{2} z^{2} \ll 1$, and expand the binomial (1 $\left.-\alpha^{2} z^{2}\right)^{-1}$ up to the first power of $(\alpha z)^{2}$. In this case the problem is equivalent to a Schrödinger equation with an inverted harmonic oscillator potential [20]. By changing variables to $z \rightarrow x \equiv \sqrt{2} \alpha z\left(2-m^{2} / \alpha^{2}\right)^{1 / 4}$, and defining $a \equiv$ $-\left(M_{5}^{2}+2 \alpha^{2}-m^{2}\right) /\left(2 \alpha \sqrt{2 \alpha^{2}-m^{2}}\right)<0$, the Schrödinger equation acquires the form

$$
\frac{d^{2}}{d x^{2}} \varphi(x)+\left(\frac{1}{4} x^{2}-a\right) \varphi(x)=0
$$

The eigenfunctions in this case are appropriate combinations of parabolic cylinder functions $W(a, x)$, which are actually oscillatory for all $x$ (since $a<0$ ) [21]. The requirement that the solution has a maximal probability density on the D3brane at $z=0$ (so as to imply some sort of localization) implies that only even functions of $x$ should be considered. The appropriate combination is $\varphi(x)=W(a, x)+W(a,-x)$, in the notation of [21]. In the regime of interest to us here $x^{2}$ $\ll-a\left(\alpha^{2} z^{2} \ll 1\right)$, which implies the asymptotic form

$$
\begin{aligned}
\varphi & =W(a, x)+W(a,-x) \\
\sim & 2 W(a, 0) e^{v_{r}(x)} \cos \left(\sqrt{-a} x+u_{i}(x)\right), \\
W(a, 0) & =2^{-3 / 4}\left|\frac{\Gamma\left(\frac{1}{4}+\frac{1}{2} i a\right)}{\Gamma\left(\frac{3}{4}+\frac{1}{2} i a\right)}\right|^{1 / 2}, \\
v_{r}(x) \sim- & \frac{(x / 2)^{2}}{(2 \sqrt{-a})^{2}}+\frac{2(x / 2)^{4}}{(2 \sqrt{-a})^{4}} \\
& -\frac{9(x / 2)^{2}+(16 / 3)(x / 2)^{6}}{(2 \sqrt{-a})^{6}}+\cdots,
\end{aligned}
$$

$$
\begin{aligned}
u_{i}(x) \sim & \frac{(2 / 3)(x / 2)^{3}}{2 \sqrt{-a}}-\frac{(x / 2)+(2 / 5)(x / 2)^{5}}{(2 \sqrt{-a})^{3}} \\
& +\frac{(16 / 3)(x / 2)^{3}+(4 / 7)(x / 2)^{7}}{(2 \sqrt{-a})^{5}}-\cdots
\end{aligned}
$$

where $v_{r}(x)$ is an even function of $x$, and $u_{i}(x)$ is an odd function of $x$. The requirement that the solution vanishes at the opaque boundaries $z= \pm 1 / \alpha,{ }^{6}$ implies the vanishing of the argument of the cosine in Eq. (29), and thus a discrete $a$-, and hence $\mathrm{KK} m$-, spectrum, for given $M_{5}, \alpha$ :

$$
\begin{aligned}
\sqrt{\frac{M_{5}^{2}+2 \alpha^{2}-m^{2}}{\alpha^{2}}}+u_{i}\left(\sqrt{2}\left(2-m^{2} / \alpha^{2}\right)^{1 / 4}\right) & \simeq\left(n+\frac{1}{2}\right) \pi, \\
n & =0,1,2, \ldots
\end{aligned}
$$

under the constraint $0<m^{2}<2 \alpha^{2}$, which restricts severely the allowed spectrum of KK modes. For instance, for $M_{5}$ $\gg \alpha$, this constraint, in conjunction with Eq. (30), implies that the allowed KK modes have $n \sim M_{5} / \alpha \gg 1$. Of course, it is understood that the above approximate analysis is strictly valid for $\alpha^{2} z^{2} \ll 1$, and should only be considered as indicative for the real case. There is one limiting case, though, where the above results are valid throughout the region 0 $<\alpha^{2} z^{2}<1$, and this is when one is dealing with nearcriticality KK modes with mass $m \rightarrow \sqrt{2} \alpha-\epsilon, \epsilon \rightarrow 0^{+}$, in the case $M_{5} \neq 0$. In this case, from Eq. (30), we observe that there is only a discrete allowed set of bulk masses $M_{5}=(n$ $\left.+\frac{1}{2}\right) \pi \alpha, n=0,1,2 \ldots$, which is compatible with the vanishing $\varphi$ boundary conditions at $z= \pm 1 / \alpha$ set by the opaque walls. The above qualitative results are supported by a numerical analysis of the complete problem including properly the boundary effects (cf. Fig. 2 for the modulus-squared of the wave functions of subcritical KK modes, which peak on the D3-brane at $z=0$ ).

A similar approximate eigenvalue analysis may be applied to KK modes with masses $m^{2}>2 \alpha^{2}$. In this case, for $|\alpha z| \ll 1$, one may deduce the approximate energyeigenvalue spectrum $E_{n}$ by analogy with the harmonic oscillator case: $E_{n} \equiv M_{5}^{2}=m^{2}-2 \alpha^{2}+\alpha \sqrt{2 m^{2}-4 \alpha^{2}}\left(n+\frac{1}{2}\right), \quad n$ $=0,1,2, \ldots$. Inverting this relation with respect to $m$ one obtains

$$
\begin{gathered}
m^{2}=2 \alpha^{2}+\frac{1}{2}\left[-\alpha\left(n+\frac{1}{2}\right)+\sqrt{2 M_{5}^{2}+\alpha^{2}\left(n+\frac{1}{2}\right)^{2}}\right]^{2}, \\
n=0,1,2, \ldots
\end{gathered}
$$

\footnotetext{
${ }^{6}$ Notice that the boundary region $z= \pm 1 / \alpha$ lies outside the regime of validity of the inverted-oscillator approximation. Nevertheless, for the qualitative description of the discrete spectrum, it is sufficient to impose the above boundary condition to the solutions of Eq. (28).
} 
We notice that the analogy with the harmonic oscillator system, with a positive minimum in the potential, implies the following restriction on KK modes (positivity of the "oscillator energy eigenvalue"'):

$$
m^{2}<M_{5}^{2}+2 \alpha^{2}
$$

It is straightforward to determine the range of $m^{2}\left(>2 \alpha^{2}\right)$ for which Eq. (32) is valid together with $M_{5}^{2} \leqslant m^{2}$ :

$$
2 \alpha^{2}<m^{2}<2 \alpha^{2}+\frac{8 \alpha^{2}}{(2 n+1)^{2}}, \quad n=0,1,2, \ldots .
$$

Outside this region, $M_{5}^{2}>m^{2}$.

Note that in standard string models one allows only the (massless) gravitational string multiplet to propagate in the bulk [2], which implies $M_{5}=0$. In view of the harmonic oscillator analogue, such a state does not exist (in a bounded form $)^{7}$ in the case $m^{2}>2 \alpha^{2}$. A numerical analysis, supported analytically by the harmonic oscillator analogy, indicates that bounded KK modes with masses $\sqrt{2} \alpha<m<M_{5}$, have wave functions that peak near (but not exactly at) the boundaries, symmetrically about the origin, but then vanish at the boundaries, due to the infinite opacity of the walls. However, for the case $M_{5}=m$, one obtains a discrete set of allowed values of $m>\sqrt{2} \alpha$ which, as demonstrated in Fig. 2, appears to exhibit localization on the D3 brane at $z=0$. This is a feature that persists in the Minkowskian treatment as we shall discuss in the next subsection. Finally, the bounded wave functions for KK modes with $m>M_{5}$, under the constraint (32), appear to have local peaks at the $z=0$ but not strong localization there (see Fig. 2). Again this feature survives the analytic continuation procedure to Minkowskian times.

Our analysis above demonstrates, therefore, that in the scenario of [6], where matter fields are also allowed to propagate in the bulk, the incorporation of stringy "recoil" (fluctuation) bulk effects of the D3-brane will imply at most a discrete $M_{5}$ mass spectrum, or, inversely, a discrete set of KK masses $m$ for given $M_{5}, \alpha$ [cf. Eqs. (30),(31)].

We now make an important comment. In the stringy problem a natural mass scale in the bulk [6] is the string scale $M_{5} \sim M_{s}$. In that case, as we have seen, a natural upper bound on $\alpha$ is a few hundreds TeV [17] in order to provide a satisfactory solution to the gauge hierarchy problem in supersymmetric models. Hence, one has a strong hierarchy of scales: $M_{s} \sim 10^{14} \mathrm{GeV} \gg \alpha \sim \mathcal{O}(100) \mathrm{TeV}$. It therefore follows that bounded KK modes with masses $m \geqslant M_{5}$ do not exist in such models, in view of the restriction (33), which, we stress, is an exclusive feature of the Euclidean treatment.

\footnotetext{
${ }^{7}$ To avoid confusion we stress, once again, that the acceptable solutions corresponding to the discrete set of "energy" eigenvalues in the analog Schrödinger problem are only the ones that are bounded, i.e., vanish at the boundaries $z= \pm 1 / \alpha$. This should always be taken properly into account in any numerical treatment (see Fig. 2).
}

Thus, one has effectively strong localization on the D3 brane at $z=0$ only of light KK modes of matter fields with masses $m \leqslant \sqrt{2} \alpha$, as in the graviton case [9]. We shall come back to this issue in the next subsection.

\section{Analytic continuation to Minkowskian space times and field localization}

In this subsection we discuss the above-described field localization after analytic continuation back to (physical) Minkowski target time. This procedure implies that in all the relevant formulas of the previous subsection there will be now a change in sign of mass-squared terms in the potential of the respective Schrödinger equations (19),(26):

$$
m^{2} \rightarrow-m^{2}, \quad M_{5}^{2} \rightarrow-M_{5}^{2} .
$$

In such a case, viewing again the $-M_{5}^{2}$ terms as energyeigenvalue terms in the corresponding Schrödinger equations, we observe that the potential at $z=0$ is attractive now for any value of $m$. Nevertheless, one obtains qualitatively the same localization behavior for the light KK modes $m$ $<\sqrt{2} \alpha$ in the massless case $M_{5}=0$ as in the corresponding Euclidean case of the previous section, as can be confirmed by a numerical analysis of the respective Schrödinger equation (see Fig. 3 where the respective probability densities are plotted). This is a nice consistency check of the Euclidean approach, which, as explained in [9], was necessitated by the requirement of convergence of the world-sheet path integrals employed in the formalism.

The case $M_{5} \neq 0$ of the Minkowskian treatment requires particular attention. As in the subcritical Euclidean case $\left(m^{2}<2 \alpha^{2}\right)$, but here for all $m$, the situation is equivalent to that of a particle bound inside a potential well (see Fig. 3), which starts as an inverted harmonic oscillator potential [20] around $z=0$, but then deviates significantly from it (by anharmonic terms), to end up in $\delta$-function opaque (repulsive) walls at $z= \pm 1 / \alpha$, with infinite opacity. Therefore the wave function should vanish at the boundaries. The energy spectrum is again discrete, and one has nodes in the wave function, whose number depends on the value of the energy $-M_{5}^{2}$ [24]. This is indeed confirmed by a numerical analysis of the Minkowskian-time version of Eq. (27) [cf. Eq. (34)].

It should be noticed that the precise behavior of the solutions in the allowed region of the bulk space, $\alpha^{2} z^{2}<1$, depends crucially on the relative magnitude of the scales $m$ and $M_{5}$ (under the appropriate constraints implied by the discreteness of the allowed energy spectrum). Specifically, for masses $M_{5}>m$, due to the shape of the potential, which dives down near the boundaries, before the $\delta$-function terms take over, the wave functions peak near the boundaries, symmetrically about the origin, as in the corresponding Euclidean case. They of course vanish at the boundaries, due to the infinite opacity of the walls (see Fig. 3). The distance between the above-mentioned peaks depends on the difference $M_{5}^{2}-m^{2}$. The two peaks meet when $M_{5}^{2} \sim m^{2}$, leading to a strong localization on the D3-brane at $z=0$ of such KK modes (see Fig. 3). As we have seen, this last feature also characterizes the Euclidean treatment. 

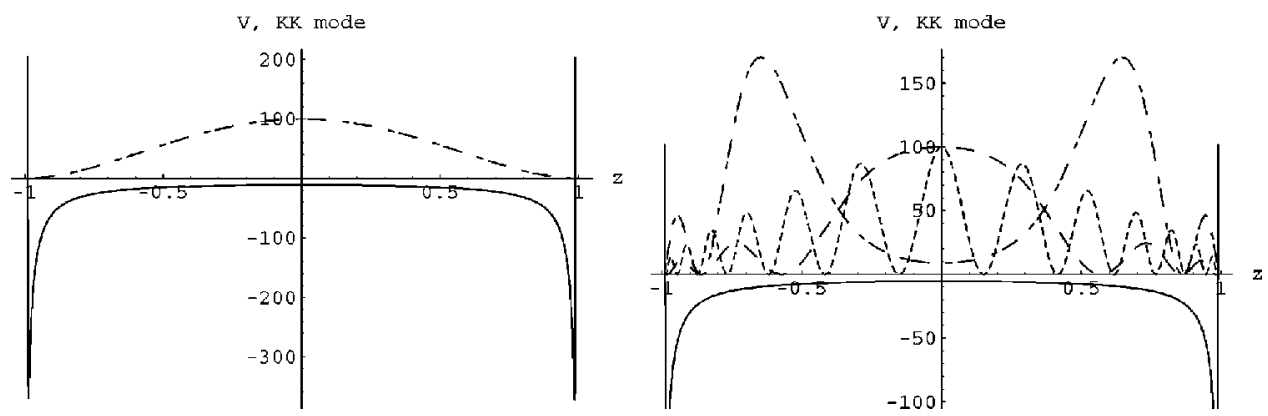

FIG. 3. Field localization in the case of Minkowskian time. The figure on the left shows the modulus squared of the massless scalar-field wave function (or, equivalently, that of a graviton mode), arbitrarily normalized, for Kaluza-Klein (KK) modes with masses below the critical mass $0 \leqslant m<m_{c r}=\sqrt{2} \alpha$ (dashed dotted curve). The wave functions corresponding to these modes are peaked on the $z=0$ brane world and vanish at the opaque boundaries. The figure on the right shows three KK modes of a massive bulk scalar field of mass $M_{5}$, with masses $m^{2}>m_{c r}^{2}$ : one with mass $m^{2} \sim M_{5}^{2}$ (dashed line), which exhibits strong localization on the D3 brane at $z=0$, one mode with mass $m^{2}<M_{5}^{2}$ (dashed dotted curve), and one mode with mass such that $m^{2}>M_{5}^{2}$ (dotted curve). The solid curves in both figures correspond to the corresponding potential of the equivalent Schrödinger equation.

Finally, in the case $m^{2}>M_{5}^{2}$, the wave function of the corresponding mode resembles that of a free particle, included in an envelope which peaks at $z=0$ and vanishes at $z= \pm 1 / \alpha$ (see Fig. 3). Thus, such KK modes are not strongly localized on the D3-brane at $z=0$, in contrast to the $M_{5}$ $=0, m<\sqrt{2} \alpha$, or $M_{5} \sim m>\sqrt{2} \alpha$ cases. Similar features have been observed for the corresponding case in the Euclidean treatment (cf. Fig. 2).

We should note, however, that in the stringy case the latter modes $\left(m>M_{5}\right)$ are superheavy, given that $M_{5} \sim M_{s}$ $\sim 10^{14} \mathrm{GeV}$. The superheavy KK modes that are allowed then in the physical low-energy spectrum are those with masses $M_{s}<m<M_{P}$ with the Planck mass $M_{P} \sim 10^{19} \mathrm{GeV}$, being viewed as a low-energy physics ultraviolet cutoff. However, consistency with the Euclidean case, for which the constraint (32) is valid, implies that $M_{0}^{2} \equiv M_{s}^{2}+2 \alpha^{2}$ is the highest allowed mass scale for the superheavy KK modes in the string-inspired model. In addition, as we mentioned at the end of the previous subsection, if one restricts the supersymmetry obstruction scale $\alpha$ to a few hundreds of $\mathrm{TeV}$ at most, motivated by a solution to the gauge hierarchy problem [17], then KK modes with $m \geqslant M_{5}$ do not exist, and one effectively obtains localization on the D3-brane of light KK modes only, with masses $m \leqslant \sqrt{2} \alpha$.

\section{BRANE QUANTUM FLUCTUATIONS IN THE RANDALL-SUNDRUM MODEL}

So far we have examined the case of a single brane, representing our world. An interesting issue is to consider the above model [9] in the context of the Randall-Sundrum model [4] involving two branes, which are assumed to be stabilized, e.g. through the mechanism of [8]. In this respect, we should stress that the non-factorizable metrics induced by quantum fluctuations of the individual branes in the RandallSundrum (RS) scenario will induce effects that are suppressed by powers of the string coupling $g_{s}$. This is due to the fact that the recoil fluctuations of the individual branes are string loop effects, as explained in detail in [11].

As a result, the induced metric (1) will appear as a small quantum correction to classical RS scenaria involving two parallel branes separated by a finite distance $r_{0}$, one of which represents the observable world. Thus, the classical Einstein's equations are no longer expected to be satisfied for the system of the two (parallel) fluctuating branes. In this sense, our quantum fluctuations play the role of inducing a scenario that is similar to the one suggested in [7]. Below we shall present it in a version [25] most suitable for our case here.

Let us consider the non-factorizable RS space-time metric, with a small perturbation $\varepsilon$ representing our quantumrecoil effects:

$$
d s^{2}=\left(e^{-2 k_{0} z}+\varepsilon\left|1-\alpha^{2} z^{2}\right|\right) \sum_{I=1}^{4} d X_{I} d X^{I}-d z^{2}
$$

where $k_{0}$ is the parameter of the RS solution, while $\varepsilon$ is assumed proportional to an appropriate power of the string coupling $g_{s} \ll 1$, as a result of the quantum nature of the recoil-induced metric. Note that, in the case of isolated D3branes examined above, such proportionality constant may be absorbed in normalization of the coordinates, but here, in view of the difference in the relative strength of the effect as compared to the classical RS solution, such factors must be kept.

In the RS scenario the bulk geometry has been restricted to the positive $z>0$ portion of the anti-de Sitter space. One may continue to the negative $z$ axis, in which case in view of the AdS conformal field theory (CFT) correspondence [26], the five-dimensional gravity theory would be equivalent to a four-dimensional conformal field theory located in the boundary of the AdS space time at $z=-\infty$. The truncated version of this space time in the RS scenario $(z>0)$ does indeed have dynamical four dimensional gravity. In a similar manner, the quantum-recoil induced space time (1), which also arises originally in a truncated version for $z>0$, due to the recoil formalism, and then is analytically continued in the form (1), as explained in [9], also gets dynamical gravity localized on the brane in the way described above. 
In the modification of the RS scenario presented in Ref. [7], and elaborated further by Witten in [25], which we adopt here, the quantum fluctuations would be unimportant as long as $e^{-k_{0} z} \gg \varepsilon$, and one would get the RS scenario. This would imply that, for four-dimensional distances not so large relative to the separation between the two branes, the world would look effectively four dimensional, due to the field localization on our brane world characterizing the conventional RS scenario [4]. In our framework, this would mean that the quantum fluctuations of the D3-brane world in the bulk would be unimportant ( $\varepsilon \rightarrow 0$, or, in our case, very weak string couplings $g_{s} \rightarrow 0$ ).

On the other hand, when one looks at four-dimensional scales sufficiently large as compared to the separation between the branes, when the quantum fluctuations of the individual branes become important, then one would expect the space time to become effectively five dimensional. However, in our quantum-fluctuating ("recoil") case, the positive energy condition, required probably for stability of the space time [25], is valid strictly inside the dynamical horizons of extent $z \sim 1 / \alpha$ on each side of a fluctuating brane. The case in which the quantum fluctuations become important would require relatively large $\varepsilon$, or, equivalently, strong string couplings $g_{s}$, and hence large $\alpha$ [cf. Eq. (2)]. This, in turn, would imply that stable space times are localized within tiny horizons around each individual brane, where the light matter fields are also localized, as we have seen above. Hence, this would prevent a possible detection of the fifth dimension by going to sufficiently large four-dimensional distances. We consider this an interesting feature of our quantum-fluctuating brane approach, which should be explored further in the context of higher-dimensional bulk space times.

\section{CONCLUSIONS}

Above we have discussed some interesting physical features of a brane-world model for the dynamical generation of a non-factorizable geometry [9] through quantum (recoil) effects, representing position and momentum fluctuations of the brane. It is important to stress that the scale of momentum fluctuations of the brane world, which are related to the position fluctuations through some stringy uncertainty relations [12], imply a mass hierarchy on the four-dimensional world. This happens without the need for implementing a second brane, and thus stabilization mechanisms, which is the case of the original Randall-Sundrum scenario [4].

If, on the other hand, one starts from the two-brane scenario of [4] and [6], then the recoil metric (1) may be viewed as a small perturbation, expressing quantum string corrections. However, even in that case, the induced space time appears to be stable (for the existence of matter) only in a small region around the fluctuating defect, thus preventing a possible detection of the fifth geometry by going to very large four-dimensional distances as claimed in [7], where the brane fluctuations are ignored.

An interesting feature of our approach is that, as a result of the bulk quantum fluctuations ("recoil") of the D3-brane, the mass spectrum of the four-dimensional KK modes is dis- crete, which seems to be a generic feature of recoil-induced space times with anti-de Sitter like bulk [23]. This comes about as a consequence of the specific form of the potential of the equivalent Schrödinger problem, which is characterized by the presence of completely impenetrable potential walls, effectively binding the analogue quantum-mechanical "particle" inside the region $\alpha^{2} z^{2}<1$. This implies an interesting phenomenology. In particular, in the context of stringinspired models, one finds that, as is the case with the light KK modes with masses $m^{2}<2 \alpha^{2}$, one also has a strong localization on the D3-brane at $z=0$ of intermediate-scale massive modes $m \sim M_{5} \sim M_{s} \sim 10^{14} \mathrm{GeV}$. Such heavy matter particles may have interesting phenomenological, as well as cosmological implications (e.g. in the cold dark-matter problem, etc).

It must be noted, however, that such modes are absent from the Euclidean time spectrum of string-inspired models in which $\alpha \leqslant 100 \mathrm{TeV} \ll M_{s} \sim 10^{14} \mathrm{GeV}$, in order to provide a solution to the conventional gauge-hierarchy problem. As stressed repeatedly in the text, the Euclidean formalism is necessitated by the requirement of convergence of the worldsheet approach to the quantum fluctuating ("recoiling") branes [9]. Hence, it seems to us, that consistency of the Minkowskian treatment with such Euclidean formalisms needs to be sought. Under this restriction, then, heavy KK states of mass $m \geqslant M_{5}$ do not exist in a bounded form, and hence only light KK modes of bulk graviton-matter fields, with four-dimensional masses $m \leqslant \sqrt{2} \alpha$, are localized on the D3-brane world at $z=0$ in such string-inspired models. However, as we stressed previously, our analysis in the present article is more general.

There are many features of the model that need to be worked out before definite conclusions are reached on realistic phenomenological issues. The most important of them is the rigorous implementation of the above formalism of recoiling (quantum fluctuating) D-branes in target-space supersymmetric theories. We have seen that the recoil will most likely obstruct supersymmetry [17], but the detailed mechanism of this obstruction and the associated mass splittings, that would affect the phenomenology of the model, have still to be worked out. Nevertheless, we feel that the features already obtained from this, rather generic, model of quantum fluctuating branes deserve further studies, especially in connection with possible experimental signatures that could be testable in future colliders or astrophysical experiments.

\section{Note added in proof}

After publication of this article an interesting article appeared [27], in which a set of consistency conditions ("sum rules") were derived from Einstein's equations for brane world scenarios with a spatially periodic internal (bulk) space. The simplest of their sum rule, their Eqs. (2.27) or (2.28), which is independent of the potential energy of the bulk matter, may be expressed simply by stating that the sum of the brane vacuum energies (tensions) must be negative or zero. This is due to the fact that this sum is bounded by a non-negative quantity, given by a (periodic) integral over the 
compact internal bulk space of the square of the derivatives of the scalar matter field with respect to the bulk coordinate.

Although in our case the internal space is not to be periodic, nevertheless it is interesting to check whether our recoil-induced metric (1), and the associated brane vacuum energy $V(z)$ in Eq. (12), referring to a "stack" of D-branes in the bulk, can satisfy this criterion. This would imply the possibility of extending our results to periodic bulk internal spaces.

It is straightforward to see in our case that the "sum" of the vacuum energies of the brane in the "stack," which characterizes our construction, is simply given by a continuous (flat) integral of $V(z)$ of Eq. (12) over the coordinate $z$ bounded in the interval $(-1 / \alpha, 1 / \alpha)$. The result turns out to be negative $\left(\varepsilon \rightarrow 0^{+}\right)$:

$$
\int_{-(1-\varepsilon) / \alpha}^{+(1-\varepsilon) / \alpha} d z V(z)=-\frac{\alpha}{2}<0,
$$

[1] A. Sen, Invited talk to be published in the Proceedings of the 29th International Conference on High-Energy Physics (ICHEP 98), Vancouver, Canada, 1998, hep-ph/9810356; J. Polchinski, Phys. Rev. Lett. 75, 4724 (1995).

[2] V.A. Rubakov and M.E. Shaposhnikov, Phys. Lett. 125B, 136 (1983); 125B, 136 (1986); K. Akama, in Lecture Notes in Physics Vol. 176 (1982), p. 267; M. Visser, Phys. Lett. 159B, 22 (1985); I. Antoniadis, Phys. Lett. B 246, 377 (1990); N. Arkani-Hamed, S. Dimopoulos, and G. Dvali, ibid. 429, 263 (1998); I. Antoniadis, N. Arkani-Hamed, S. Dimopoulos, and G. Dvali, ibid. 436, 257 (1998).

[3] E.G. Floratos and G.K. Leontaris, Phys. Lett. B 465, 95 (1999); A. Kehagias and K. Sfetsos, ibid. 472, 39 (2000); D. J. Chung, L. Everett, and H. Davoudiasl, Phys. Rev. D (to be published), hep-ph/0010103.

[4] L. Randall and R. Sundrum, Phys. Rev. Lett. 83, 3370 (1999); 83, 4690 (1999).

[5] Some selected references are M. Gogberashvili, hep-ph/9812296; M. Gogberashvili, Europhys. Lett. 44, 396 (2000); A. Kehagias, Phys. Lett. B 469, 123 (1999); J. Lykken and L. Randall, J. High Energy Phys. 06, 014 (2000); C. Csaki, M. Graesser, C. Kolda, and J. Terning, Phys. Lett. B 462, 34 (1999); T. Nihei, ibid. 465, 81 (1999); N. Kaloper, ibid. 474, 269 (2000); I. Oda, ibid. 480, 305 (2000); 472, 59 (2000); A. Brandhuber and K. Sfetsos, J. High Energy Phys. 10, 013 (1999); K. Behrndt and M. Cvetic, Phys. Lett. B 475, 253 (2000); H. Davoudiasl, J.L. Hewett, and T.G. Rizzo, ibid. 473, 43 (2000); Phys. Rev. Lett. 84, 2080 (2000); P. Kanti, I. Kogan, K. Olive, and M. Pospelov, Phys. Lett. B 468, 31 (1999); P. Binetruy, C. Deffayet, U. Ellwanger, and D. Langlois, ibid. 477, 285 (2000); C. Csaki, J. Erlich, T. J. Hollowood, and Y. Shirman, Nucl. Phys. B581, 309 (2000); I. I. Kogan, S. Mouslopoulos, A. Papazoglou, and G. G. Ross, ibid. B584, 313 (2000); H. Hatanaka et al., Prog. Theor. Phys. 102, 1213 (1999); S. Nam, J. High Energy Phys. 04, 002 (2000); N. Kaloper, J. March-Russell, G. D. Starkman, and M. Trodden, Phys. Rev. Lett. 85, 928 (2000); C. Csaki, J. Erlich, C. under proper treatment of the boundary singularities which cancel out. This implies, therefore, that the sum rule (2.27) or (2.28) of [27] is satisfied in our scenario. Thus, in principle, our construction, which takes proper account of the back reaction of matter on the bulk geometry surrounding the recoiling brane, can be extended to periodic internal spaces, as expected.

\section{ACKNOWLEDGMENTS}

This work is partially supported by the European Union (contract Ref. HPRN-CT-2000-00152). Parts of it have been presented (by N.E.M.) at the HEP2000 International Workshop of the Hellenic Society for High Energy Physics, Ioannina, Greece. We thank the organizers of that meeting for their interest in our work. N.E.M. also wishes to thank H. Hofer for his interest and support.
Grojean, and T. Hollowood, Nucl. Phys. B584, 359 (2000); S. Kachru, M. Schulz, and E. Silverstein, Phys. Rev. D 62, 045021 (2000); C. Csaki, J. Erlich, and T. J. Hollowood, Phys. Lett. B 481, 107 (2000).

[6] W.D. Goldberger and M.B. Wise, Phys. Rev. D 60, 107505 (1999).

[7] R. Gregory, V. A. Rubakov, and S. M. Sibiryakov, Phys. Rev. Lett. 84, 5928 (2000)

[8] W. D. Goldberger and M. B. Wise, Phys. Rev. Lett. 83, 4922 (1999); Phys. Lett. B 475, 275 (2000).

[9] G. K. Leontaris and N. E. Mavromatos, Phys. Rev. D 61, 124004 (2000).

[10] I. I. Kogan, N. E. Mavromatos, and J. F. Wheater, Phys. Lett. B 387, 483 (1996).

[11] J. Ellis, N. E. Mavromatos, and D. V. Nanopoulos, Int. J. Mod. Phys. A 13, 1059 (1998); J. Ellis, P. Kanti, N. E. Mavromatos, D. V. Nanopoulos, and E. Winstanley, Mod. Phys. Lett. A 13, 303 (1998); for a concise review, see J. Ellis, N. E. Mavromatos, and D. V. Nanopoulos, Proceedings of the 4th International Symposium on Sources and Detection of Dark Matter in the Universe (DM 2000), Marina del Rey, California, 2000, gr-qc/0005100, and references therein.

[12] N. E. Mavromatos and R. J. Szabo, Phys. Rev. D 59, 104018 (1999).

[13] V. Gurarie, Nucl. Phys. B410, 535 (1993); J.S. Caux, I.I. Kogan, and A.M. Tsvelik, ibid. B466, 444 (1996); M.A.I. Flohr, Int. J. Mod. Phys. A 11, 4147 (1996); M.R. Gaberdiel and H.G. Kausch, Nucl. Phys. B489, 293 (1996); I.I. Kogan and N.E. Mavromatos, Phys. Lett. B 375, 111 (1996); M.R. Rahimi-Tabar, A. Aghamohammadi, and M. Khorrami, Nucl. Phys. B497, 555 (1997); I.I. Kogan, A. Lewis, and O.A. Soloviev, Int. J. Mod. Phys. A 13, 1345 (1998); I.I. Kogan and J.F. Wheater, Phys. Lett. B 486, 353 (2000); A. Lewis, hep-th/0009096.

[14] M. Li and T. Yoneya, Phys. Rev. Lett. 78, 1219 (1997), and references therein.

[15] R.M. Wald, General Relativity (University of Chicago Press, 
Chicago, 1984); in the present context, see D. Z. Freedman, S. S. Gubser, K. Pilch, and N. P. Warner, Adv. Theor. Math. Phys. 3, 363 (1999).

[16] J. Ellis, N. E. Mavromatos, and D. V. Nanopoulos, Phys. Rev. D 62, 084019 (2000).

[17] A. Campbell-Smith and N. E. Mavromatos, Phys. Lett. B 488, 199 (2000).

[18] E. Witten, Int. J. Mod. Phys. A 10, 1247 (1995).

[19] I. Antoniadis and B. Pioline, Nucl. Phys. B550, 41 (1999).

[20] G. Barton, Ann. Phys. (N.Y.) 166, 322 (1986).

[21] Handbook of Mathematical Functions, edited by M. Abramowitz and I. A. Stegun, Nat. Bur. Stand., Appl. Math. Ser. 55, (U.S. GPO, Washington, DC, 1964).

[22] J. A. Stratton, Proc. Natl. Acad. Sci. U.S.A. 21, 51 (1935); 21, 316 (1935); E. Fisher, Philos. Mag. 24 (7), 245 (1937); E.
Kamke, Differentialgleichungen, Lösungsmethoden und Lösungen (Akademische Verlagsgesellschaft, Geest \& Portig K.G., Leipzig, 1959); G. Latta, J. Math. Phys. 4, 139 (1963).

[23] N. E. Mavromatos and E. Winstanley, Int. J. Mod. Phys. A 16, 251 (2001).

[24] See, for instance, G. Birkoff and G.-C. Rotta, Ordinary Differential Equations (Wiley, New York, 1959); A. Messiah, Quantum Mechanics (North-Holland, Amsterdam, 1970), Vol. I.

[25] E. Witten, Proceedings of the 4th International Symposium on Sources and Detection of Dark Matter in the Universe (DM 2000), Marina del Rey, California, 2000, hep-ph/0002297.

[26] J. Maldacena, Adv. Theor. Math. Phys. 2, 231 (1998).

[27] G. Gibbons, R. Kallosh, and A. Linde, J. High Energy Phys. 01, 022 (2001). 